\title{
Verklebung von wachsimprägnierter Buche unter Variation der Klebesysteme und Durchführung einer Plasmabehandlung
}

\author{
G. Scholz • E. Nothnick • G. Avramidis • A. Krause • \\ H. Militz · W. Viöl · A. Wolkenhauer
}

Eingegangen: 28. April 2010 / Online publiziert: 22. Juli 2010

(C) The Author(s) 2010. Dieser Artikel ist auf Springerlink.com mit Open Access verfügbar

Zusammenfassung Buchenholz wurde mit heiß schmelzendem Wachs imprägniert. Durch Kontaktwinkelmessungen wurde der hydrophobe Charakter des imprägnierten Holzes quantifiziert. Mit Zugscherversuchen wurden die Verklebungsgüte nach verschiedenen Lagerungsfolgen geprüft. Wachsimprägnierte Buche wurde einer Plasmabehandlung unterzogen und ihre Wirkung auf die Verklebungsgüte untersucht. Das unpolare Paraffin zeigte schlechtere Verklebungsresultate als das Montanesterwachs, so dass ein primärer Einfluss des verwendeten Wachstyps auf die Verklebung des imprägnierten Holzes deutlich wird. Eine Verklebung imprägnierter Buche mit Montanesterwachs ist im Innenbereich für konstruktive Holzbauteile möglich. Unter dauerfeuchten Bedingungen werden durch konventionelle, mit Härtern versehene Polyvinylacetat-Leime und PhenolResorcinol-Harz D3 Eigenschaften im nichtkonstruktiven Bereich erreicht. Die Plasmabehandlung verbessert diese Verklebungseigenschaften auf D4. Im wechselfeuchten Um-

This article is dedicated to Gerd Wegener on the occasion of his retirement as professor at the Technische Universität München.

G. Scholz $(\bowtie) \cdot$ A. Krause $\cdot$ H. Militz

Holzbiologie und Holzprodukte, Georg-August-Universität

Göttingen, Büsgenweg 4, 37077 Göttingen, Deutschland

e-mail: gscholz@gwdg.de

H. Militz

e-mail: hmilitz@gwdg.de

E. Nothnick · G. Avramidis · W. Viöl · A. Wolkenhauer Naturwissenschaften und Technik, Hochschule für Angewandte Wissenschaft und Kunst, Von-Ossietzky-Strasse 99, 37085 Göttingen, Deutschland

W. Viöl · A. Wolkenhauer

Laser-Laboratorium Göttingen e.V., Hans-Adolf-Krebs-Weg 1,

37077 Göttingen, Deutschland gebungsbereich bewährten sich Isocyanatkleber und nach einer Plasmabehandlung von 120 s Polyvinylacetat-Leim.

\section{Adhesion of wax impregnated solid beech wood with different glues and by plasma treatment}

Abstract Beech wood was impregnated with two hot melting waxes. Contact angle measurement followed to determine hydrophobizing of such treated wood. Tensile shear tests were carried out to test the adhesion properties with three different glues under varying environmental conditions. Furthermore, wax impregnated beech was subjected to plasma treatment prior to glueing. The results demonstrate that wood treated with polar paraffin shows lower adhesion properties compared to montan wax. Therefore, montan wax was investigated more intensely. Beech impregnated with such wax can be used for constructive building elements in the interior sector. D3 Glues for non-constructive applications were realized by using PVAc and phenol-resorcinol resin under permanent influence of humidity. Plasma treatment improves this quality to obtain D4 glue. Back-drying of the water stored specimens proves the suitability of isocyanate glue and also that of PVAc after plasma treatment for $120 \mathrm{~s}$.

\section{Einleitung}

Die Hydrophobierung von Holz dient der Reduzierung der Wasseraufnahme mit dem Ziel, die Entstehung von Rissen und Verwerfungen oder die Auswaschung phenolischer Zersetzungsprodukte des UV-Abbaus zu verringern. Die Verwendung von Wachsen als Hydrophobierungsmittel wird beispielsweise von Borgin und Corbett (1970), Sell (1977), Feist und Mraz (1978) oder Scholz et al. (2009) beschrieben.

Wachse sind Ester langkettiger Fettsäuren mit höheren Alkoholen (Fengel und Wegener 2003). Nach Illmann et 
al. (1983) besitzen verschiedene synthetische Stoffe wie Polyolefine oder langkettige Alkane wie Fischer-TropschWachse wachsartige technische und physikalische Eigenschaften. Aufgrund dieser Eigenschaften wie Schmelzpunkt oder temperaturabhängige Viskosität werden sie von der Deutschen Gesellschaft für Fettwissenschaft als Wachse eingestuft (DGF-Einheitsmethoden 1975). Die wasserabweisenden Eigenschaften erschweren allerdings die Verklebung modifizierten und hydrophobierten Holzes, da viele konventionelle Leime wasserbasiert sind. Diese Erscheinung ist auch für einige tropische Kernhölzer typisch, die höhere Gehalte hydrophober Substanzen aufweisen, wohingegen höhere Phenolgehalte bindungsfördernd wirken (Harzmann 1988).

Ein weiterer, bei der Holzimprägnierung mit heiß schmelzenden Wachsen, auftretender Effekt ist die mechanische Blockierung der Lumen durch eingelagertes Wachs und eine damit einhergehende Verkleinerung der für die Haftung zur Verfügung stehenden Oberfläche. Scholz et al. (2010a) beschreiben den Einfluss verschiedener Wachstypen auf die Holzfestigkeit. Der Faserverbund kann im Hinblick auf einen Holzbruch durch eine Wachsbehandlung verstärkt werden. In Abhängigkeit der Viskosität und Polarität des verwendeten Wachses können Scholz et al. (2010b) unterschiedliche Eindringtiefen in das Holz nachweisen. Auch die Schrumpfung beim Erkalten ist wachsspezifisch, so dass dabei entstehende Hohlräume verschiedenartig beschaffen sein können. Ein Einfluss des Wachstyps auf die Verklebbarkeit wurde deshalb nicht ausgeschlossen.

Militz und Krause (2008) erzielten für wachsimprägnierte Kiefer unter variierenden Umgebungsbedingungen gute Verklebungsergebnisse mit Melamin-Harnstoff-Formaldehyd in Kombination mit einem Härter. Kurt et al. (2008) konnten eine Steigerung der Zugscherfestigkeiten wachsimprägnierter Kiefer durch hydroxymethyliertes Resorcin erreichen, wobei unter feuchten Umgebungsbedingungen die Ergebnisse signifikant unter denen der Kontrollen lagen.

Im vorliegenden Artikel wurde der Einfluss von polarem Montanesterwachs und unpolarem Fischer-TropschWachs auf die Verklebung untersucht. Neben unterschiedlichen Wachsen und Verleimungssystemen wurde auch der Einfluss einer Plasmabehandlung unter Atmosphärendruck auf die Verklebungsqualität untersucht. Der positive Einfluss einer Plasmabehandlung auf die Oberflächen- und Verklebeeigenschaften von Holz und Holzwerkstoffen konnte bereits in vielen Untersuchungen nachgewiesen werden (Wolkenhauer et al. 2008a, 2008b, 2008c, 2009; Wolkenhauer 2009; Avramidis et al. 2010a). Da eine Plasmabehandlung die Oberflächenhydrophilität steigert und damit die Benetzung und Haftung von wässrigen Systemen fördert, könnten die Verklebeeigenschaften des durch die Wachbehandlung hydrophobierten Buchenholzes verbessert werden. Diese Steigerung der Oberflächenhydrophilität durch eine Plasmabe- handlung von wachsimprägniertem Buchenholz konnte bereits in einer vorherigen Untersuchung bestätigt werden und stellt eine Verbesserung der Verklebeeigenschaften in Aussicht (Avramidis et al. 2010b).

\section{Material und Methoden}

\subsection{Wachsbehandlung}

Buche (Fagus sylvatica L.) mit einer Jahrringlage zwischen 0 und $45^{\circ}$ wurde darrgetrocknet. Anschließend erfolgte die Vollimprägnierung mit dem Montanesterwachs Licowax E (Clariant) bzw. aus dem Fischer-Tropsch-Prozess stammenden Paraffin Paraflint C80 (Sasol) unter Vakuum (0,1 bar für $60 \mathrm{~min}$ ) und Druck (12 bar für $120 \mathrm{~min}$ ) bei $100^{\circ} \mathrm{C}$. Nach der Vollimprägnierung wurden die Proben $(380 \times 110 \times$ $10 \mathrm{~mm}^{3}$ ) bei $20^{\circ} \mathrm{C}$ und $65 \%$ relativer Luftfeuchte gelagert.

\subsection{Kontaktwinkelmessung}

Für die Kontaktwinkelmessungen (liegender Tropfen, $V=10 \mu \mathrm{l}$ ) wurden die Flüssigkeiten Diiodmethan, Ethylenglycol, Formamid, Glycerol und destilliertes Wasser verwendet. Die Messung erfolgt mit dem Contact Angle Measuring System G10 und der dazugehörigen Software DSA der Firma Krüss. Für jede zu untersuchende Oberfläche werden die 10 Kontaktwinkel der fünf genannten Flüssigkeiten nach der CWRA-Methode (constant wetting rate method) (Nussbaum 1999) ermittelt und anschließend die Oberflächenenergien nach Owens-Wendt errechnet (Owens und Wendt 1969).

\subsection{Verklebung}

Die Prüfkörper wurden nach EN 302-1 (2004) aus den gehobelten Proben angefertigt und geprüft. Für die Verleimung wurden die in Tab. 1 aufgeführten Kleber und dazugehörigen Härter verwendet. Die Prüfung erfolgte nach Beanspruchung

A1 7 Tage Lagerung im Normalklima $\left(20^{\circ} \mathrm{C}\right.$; $65 \%$ rel. Feuchte)

A2 7 Tage Lagerung im Normalklima; 4 Tage Wasserlagerung $\left(20^{\circ} \mathrm{C}\right)$

A3 7 Tage Lagerung im Normalklima; 4 Tage Wasserlagerung $\left(20^{\circ} \mathrm{C}\right) ; 7$ Tage Normalklima.

\subsection{Plasmabehandlung}

Die Plasmabehandlung wurde mittels einer dielektrisch behinderten Entladung (DBD) unter Atmosphärendruck durchgeführt. Die zu behandelnden Proben werden zwischen einer Hochspannungselektrode und einer geerdeten Gegenelektrode (Dielektrikum: Glas, $5 \mathrm{~mm}$ ) positioniert 
Tab. 1 Verwendete Klebstoffe und Härter

Table 1 Adhesives and hardeners used

\begin{tabular}{lllll}
\hline Bezeichnung & Art & Hersteller & Kleber & Härter \\
\hline EPI & Copolymeres Isocyanat & Jowat & Jowacoll 102.49 & Jowat 195.60 (10\%) \\
PVAc & Polyvinylacetat & Jowat & Jowacoll 102.26 & Jowat 195.35 (5\%) \\
PH & Phenol-Resorcinol Harz & Dynea & Aeroduc 185 & HRP 150 (17\%) \\
\hline
\end{tabular}

\begin{tabular}{lllr}
\hline Oberflächenenergie & $\begin{array}{l}\text { Gesamt } \\
\left(\mathrm{mN} \mathrm{m}^{-1}\right)\end{array}$ & $\begin{array}{l}\text { Dispers } \\
\left(\mathrm{mN} \mathrm{m}^{-1}\right)\end{array}$ & $\begin{array}{r}\text { Polar } \\
\left(\mathrm{mN} \mathrm{m}^{-1}\right)\end{array}$ \\
\hline Referenz & $55,5 \pm 0,8$ & $42,4 \pm 0,4$ & $13,0 \pm 0,4$ \\
Montanesterwachs & $29,6 \pm 0,4$ & $28,9 \pm 0,3$ & $0,6 \pm 0,1$ \\
Montanesterwachs + 4 s Plasma & $45,2 \pm 0,4$ & $29,4 \pm 0,1$ & $15,8 \pm 0,3$ \\
Montanesterwachs + 120 s Plasma & $51,2 \pm 0,7$ & $27,1 \pm 0,3$ & $24,0 \pm 0,4$ \\
Fischer-Tropsch Wachs & $27,7 \pm 0,5$ & $27,7 \pm 0,4$ & $0,02 \pm 0,01$ \\
Fischer-Tropsch Wachs + 4 s Plasma & $36,9 \pm 0,4$ & $24,2 \pm 0,2$ & $12,7 \pm 0,3$ \\
Fischer-Tropsch Wachs + 120 s Plasma & $46,1 \pm 0,4$ & $24,9 \pm 0,2$ & $21,1 \pm 0,3$ \\
\hline
\end{tabular}

Tab. 2 Oberflächenenergien unbehandelter und wachsimprägnierter Buche ohne und mit Plasmabehandlung

Table 2 Surface energies of untreated and wax-impregnated beech without and with plasma treatment

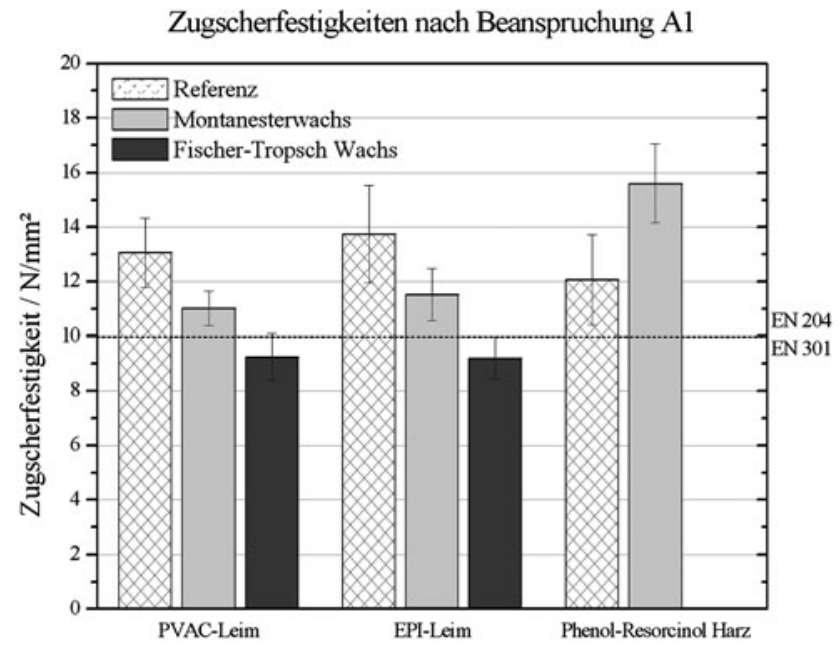

Abb. 1 Zugscherfestigkeiten aller Leime nach Beanspruchung A1 (für das Fischer-Tropsch-Wachs wurden keine Versuche mit PH durchgeführt)

Fig. 1 Tensile shear strengths for all tested adhesives according to the conditions of A1 (for the Fischer-Tropsch-wax no tests were carried out with $\mathrm{PH}$ adhesive)

keit des verwendeten Klebers. In EN 301 (2006) für tragende sowie EN 204 (2001) für nichttragende Holzbauteile wird bei Beanspruchung nach A1 eine mittlere Zugscherfestigkeit von $10 \mathrm{~N} \mathrm{~mm}^{-2}$ gefordert. Die unbehandelten Referenzen sowie die mit Montanesterwachs imprägnierten Proben zeigen für alle verwendeten Kleber eine Überschreitung dieser erforderlichen Zugscherfestigkeit. Diese wird von den mit Paraffin imprägnierten Proben nicht erreicht, wodurch diese Proben für den Einsatz als tragende Holzbauteile im Innenbereich ungeeignet sind.

Während der Anteil des Holzbruchs bei den Referenzen je nach verwendetem Kleber zwischen $66-81 \%$ lag, prägnierter sowie nichtimprägnierter Proben in Abhängig- 
Tab. 3 Leimbruchanteil in Abhängigkeit von Behandlungsart, Verklebung und Beanspruchung

Table 3 Adhesive breakage rate in dependence of treatment, glueing and test method

\begin{tabular}{|c|c|c|c|c|c|c|c|c|c|c|}
\hline \multirow[t]{2}{*}{ Typ } & \multirow{2}{*}{$\begin{array}{l}\text { Plasma } \\
\text { (s) }\end{array}$} & \multicolumn{3}{|l|}{ EPI } & \multicolumn{3}{|c|}{ PVAC } & \multicolumn{3}{|l|}{$\mathrm{PH}$} \\
\hline & & $\overline{\mathrm{A} 1}$ & A2 & $\mathrm{A} 3$ & $\overline{\mathrm{A} 1}$ & A2 & $\mathrm{A} 3$ & $\overline{\mathrm{A} 1}$ & A2 & A3 \\
\hline \multirow{3}{*}{$\begin{array}{l}\text { Montan- } \\
\text { esterwachs }\end{array}$} & - & 91 & 100 & 58 & 100 & 100 & 78 & 72 & 83 & 94 \\
\hline & 4 & - & 100 & 96 & - & 100 & 78 & - & 70 & 70 \\
\hline & 120 & - & 100 & 80 & - & 100 & 75 & - & 71 & 71 \\
\hline Paraffin & - & 100 & - & - & 100 & - & - & - & - & - \\
\hline Kontrolle & - & 34 & 100 & 12 & 19 & 86 & 4 & 21 & 36 & 30 \\
\hline
\end{tabular}

sank er bei wachsimprägnierten Prüfkörpern auf einen Holzbruchanteil zwischen 0-28 \% (Tab. 3). Als Ursache hierfür ist die Verkleinerung der für die Verklebung zur Verfügung stehenden Holzoberfläche infolge der physikalischen Abdichtung der Lumen durch die Wachsdepositen zu sehen. Nach Suchsland (1958) besteht Holz durch angeschnittene Kapillaren aus einer wellenförmigen Oberfläche. Bei unbehandeltem Holz können zusätzlich die Innenflächen zu einer Festigkeitserhöhung der Verleimung beitragen. Diese Flächen stehen jedoch den zum Teil hochviskosen Klebern durch wachsgefüllte Kapillaren nicht mehr zur Verfügung. Bei wasserbasierten Klebern erschwert zusätzlich der Hydrophobierungseffekt des Wachses die Verklebung.

\subsection{Beanspruchung für dauerfeuchte Bereiche (A2)}

In den Abb. 2a, 2b, 2c sind die Ergebnisse der Tests nach Beanspruchung im dauerfeuchten Bereich aufgeführt. Die Vorversuche zur Verklebung von mit dem Fischer-TropschWachs behandeltem Holz verliefen für die Beanspruchung A2 und A3 auch mit Plasmabehandlung unbefriedigend. Deshalb wird im Folgenden nur noch die Verleimungsgüte von mit Montanesterwachs imprägniertem Buchenholz dargestellt.

\subsubsection{PVAC-Leim}

Bei den mit PVAC-Leim verklebten Proben kann eine Abnahme der Zugscherfestigkeit um ca. 50\% infolge der Wachsimprägnierung festgestellt werden. Dabei wird die Zugscherfestigkeit auf einen Wert unterhalb der nach EN 301 für tragende Bauteile geforderten mittleren Zugscherfestigkeit von $6 \mathrm{~N} \mathrm{~mm}^{-2}$ herabgesetzt. Die Anforderungen an einen D4-Leim für nichttragende Bauteile (EN 204) können ebenfalls nicht erfüllt werden. Eine Plasmabehandlung von $4 \mathrm{~s}$ zeigt keine signifikante Beeinflussung der Zugscherfestigkeit der imprägnierten Proben. Lediglich die Anforderungen an einen D3 Leim werden erfüllt. Eine Behandlungszeit von $120 \mathrm{~s}$ führt zu einer Erhöhung der Zugscherfestigkeit, wodurch die Anforderungen der EN 204 an einen D4-Leim erfüllt werden. Der Einsatz als tragende Bauteile ist jedoch auch für diese Proben nicht möglich.

\subsubsection{EPI-Leim}

Entsprechend den Zugscherfestigkeitsprüfungen mit PVACLeim verklebten Proben kann für EPI-verleimte Prüflinge eine Reduzierung der Zugscherfestigkeit um ca. 50\% festgestellt werden (Abb. 3). Eine Plasmabehandlung der imprägnierten Proben zeigt hier jedoch auch nach einer Behandlungszeit von 120 s keine signifikante Beeinflussung der Zugscherfestigkeit. Obwohl die Mittelwerte der imprägnierten sowie imprägnierten und anschließend plasmabehandelten Proben mit über $2 \mathrm{~N} \mathrm{~mm}^{-2}$ der mittleren Zugscherfestigkeit eines D3-Leims für nichttragende Holzbauteile entsprechen (EN 204), muss dieser Einsatzbereich aufgrund der hohen Standardabweichungen kritisch betrachtet werden. Die Anforderungen an einen D4Leim nach EN 204 werden durch die Referenzen erfüllt.

\subsubsection{PH-Leim}

Abb. 2c zeigt die Ergebnisse, der mit Phenol-Resorcinol Harz verklebten Proben. Bei allen Testvarianten wird die nach EN 301 für tragende Bauteile erforderliche Zugscherfestigkeit von $6 \mathrm{~N} \mathrm{~mm}^{-2}$ erreicht, wobei nur wachsimprägnierte sowie imprägnierte und $4 \mathrm{~s}$ plasmabehandelte Proben aufgrund hoher Standardabweichungen in diesem Bereich kritisch betrachtet werden müssen. Die Anforderungen der EN 204 für nichttragende Bauteile werden dahingegen von allen Testreihen erfüllt. Während die Wasserlagerung bei PVAc und EPI zu vollständigem Leimbruch führt, wird dieser bei dem PH-Kleber auf 70-83\% gesenkt (Tab. 3).

\subsection{Beanspruchung in wechselfeuchten Bereichen (A3)}

In den Abb. 3a, 3b, 3c sind die Ergebnisse der Zugscherprüfungen nach Beanspruchung im wechselfeuchten Bereich dargestellt.

\subsubsection{PVAC-Leim}

Mit PVAC-Leim verklebte Prüfkörper zeigen eine deutliche Reduzierung der Zugscherfestigkeit infolge der Imprägnie- 


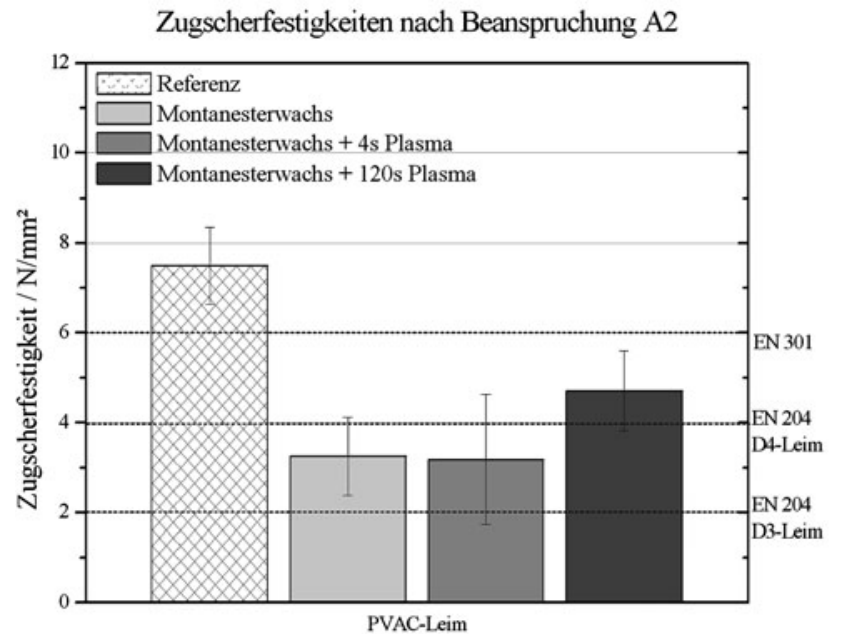

(a)

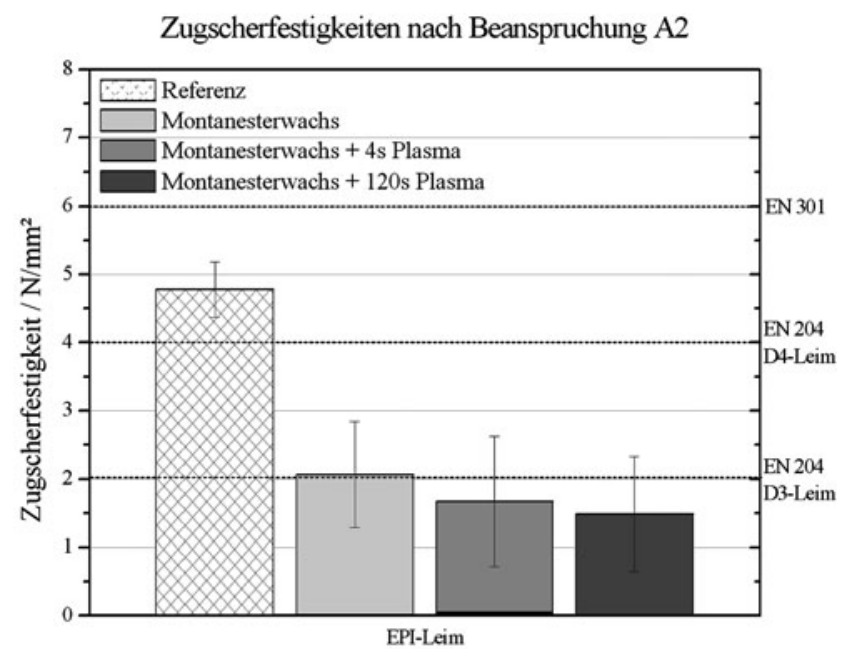

(b)

Zugscherfestigkeiten nach Beanspruchung A2

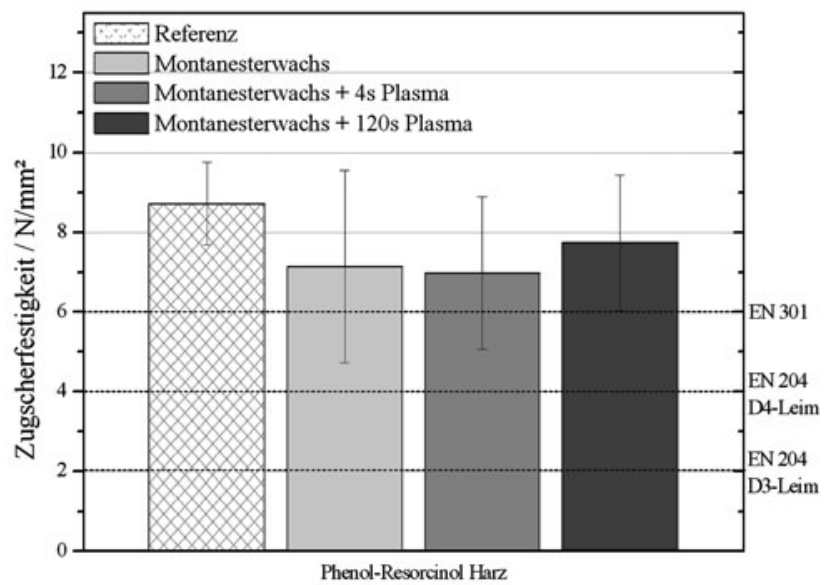

(c)

Abb. 2 (a) Zugscherfestigkeit PVAC-Leim verklebter Proben nach Beanspruchung A2. (b) Zugscherfestigkeit EPI-Leim verklebter Proben nach Beanspruchung A2. (c) Zugscherfestigkeit PH-Leim verklebter Proben nach Beanspruchung A2

Fig. 2 (a) Tensile shear strengths for specimens, glued with PVAC adhesive according to the conditions of A2. (b) Tensile shear strengths for specimens, glued with EPI adhesive according to the conditions of A2. (c) Tensile shear strengths for specimens, glued with PH adhesive according to the conditions of A2

rung mit Montanesterwachs. Durch eine Plasmabehandlung kann diese jedoch wieder erhöht werden, wobei die Steigerung der Zugscherfestigkeit mit der Behandlungsdauer zunimmt.

Die Mittelwerte aller untersuchten Testreihen liegen über dem nach EN 301 für tragende Holzkonstruktionen geforderten Mindestwert von $8 \mathrm{~N} \mathrm{~mm}^{-2}$, wobei die imprägnierten sowie die imprägnierten und anschließend $4 \mathrm{~s}$ plasmabehandelten Proben aufgrund ihrer hohen Standardabweichungen für dieses Einsatzgebiet nicht geeignet sind. Für den Einsatz einer PVAC-Leim Verklebung als D3Leim für nichttragende Holzkonstruktionen nach EN 204 ist ebenfalls eine Zugscherfestigkeit von $8 \mathrm{~N} \mathrm{~mm}^{-2}$ erforderlich.

\subsubsection{EPI-Leim}

Bei den mit EPI-Leim verklebten Proben ist eine Reduzierung der Zugscherfestigkeit infolge der Imprägnierung mit dem Montanesterwachs erkennbar. Eine Plasmabehandlung der imprägnierten Proben wirkt sich nicht signifikant auf die Zugscherfestigkeit aus.

Die Mittelwerte aller Testreihen der mit EPI-Leim verklebten Proben liegen über der nach EN 301 für tragende Holzbauteile erforderlichen Zugscherfestigkeit von $8 \mathrm{~N} \mathrm{~mm}^{-2}$, wobei sich die imprägnierten und anschließend plasmabehandelten Proben aufgrund der hohen Standardabweichungen nicht für dieses Einsatzgebiet eignen. Die Anforderungen der EN 204 an eine D3-Leim Verklebung für 


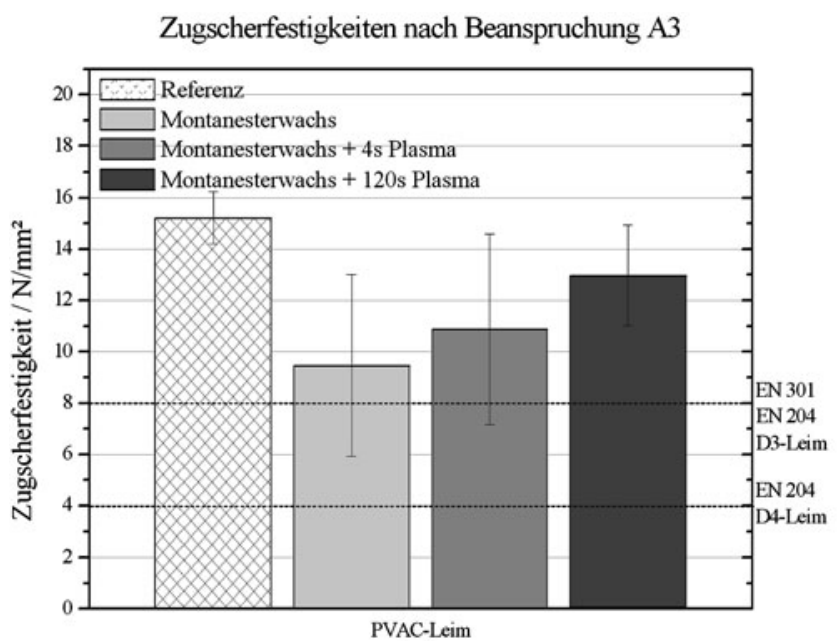

(a)
Zugscherfestigkeiten nach Beanspruchung A3

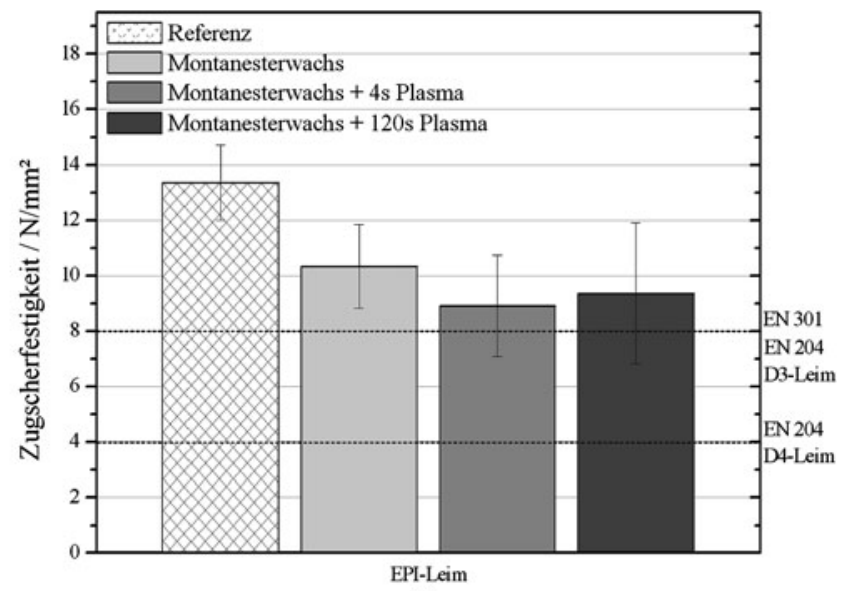

(b)

Zugscherfestigkeiten nach Beanspruchung A3

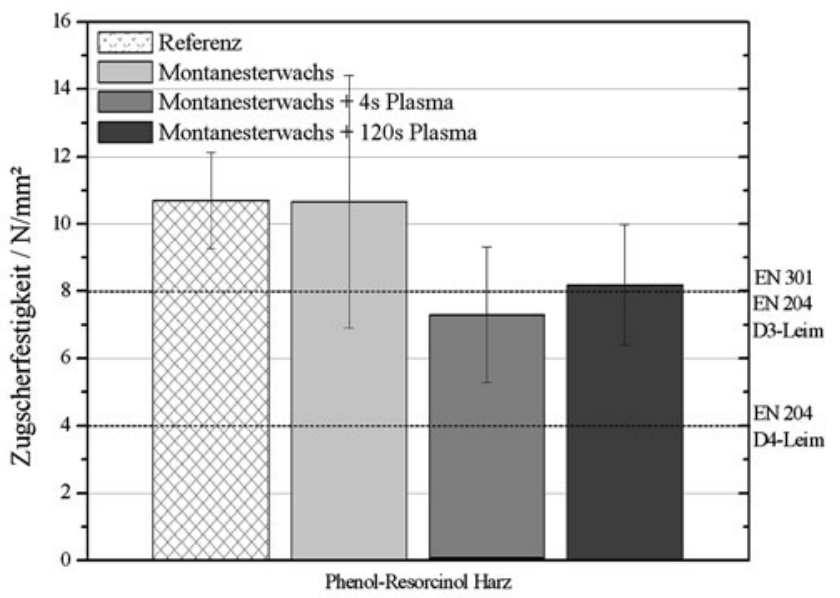

(c)

Abb. 3 (a) Zugscherfestigkeit PVAC-Leim verklebter Proben nach Beanspruchung A3. (b) Zugscherfestigkeit EPI-Leim verklebter Proben nach Beanspruchung A3. (c) Zugscherfestigkeit PH-Leim verklebter Proben nach Beanspruchung A3

Fig. 3 (a) Tensile shear strengths for specimens, glued with PVAC adhesive according to the conditions of A3. (b) Tensile shear strengths for specimens, glued with EPI adhesive according to the conditions of A3. (c) Tensile shear strengths for specimens, glued with PH adhesive according to the conditions of A3

nichttragende Holzkonstruktionen werden ebenfalls ab einer Zugscherfestigkeit von $8 \mathrm{~N} \mathrm{~mm}^{-2}$ erfüllt.

\subsubsection{PH-Leim}

Bei den mit Phenol-Resorcinol Harz verklebten Proben ist bezüglich des Mittelwertes keine Beeinflussung der Zugscherfestigkeit durch die Imprägnierung mit Montanesterwachs erkennbar. Die wachsimprägnierten Proben weisen jedoch eine deutlich erhöhte Standardabweichung sowie einen höheren Leimbruchanteil als die Referenzproben auf. Aufgrund der großen Standardabweichungen sind diese Proben nach EN 301 sowie den Anforderungen der EN 204 an eine D3-Leim-Verklebung als tragende und nichttragende Bauteile ungeeignet. Eine Plasmabehandlung der impräg- nierten Proben führt zu einer Reduzierung der Zugscherfestigkeit um ca. $30 \%$, wodurch auch diese Proben den Anforderungen der EN 301 sowie EN 204 nicht genügen.

\section{Schlussbetrachtung}

Die Verklebung von mit Wachs vollimprägniertem Buchenholz ist grundsätzlich möglich. Es zeigt sich, dass neben der Auswahl eines geeigneten Klebers auch der Wachstyp von Bedeutung für die Zugscherfestigkeit ist. Bei Vollimprägnierung von Buche mit Montanesterwachs wurden trotz vorwiegenden Leimbruchs folgende Ergebnisse erzielt:

Im Innenbereich (EN 301 2006, A1) ist bei der Verklebung wachsimprägnierter Buche die Leimart für die Ge- 
währleistung ausreichender Festigkeiten, auch für konstruktive Belastungen, nicht von Bedeutung. Die von der Norm geforderten Mindestwerte wurden in jedem Fall überschritten.

Im dauerfeuchten Bereich (EN 301 2006, A2) werden für wachsimprägniertes Holz, welches mit PVAC-Leim verklebt ist, die Anforderungen eines D3 Leimes für nichttragende Holzkonstruktionen erfüllt. Nach einer Plasmabehandlungszeit von $120 \mathrm{~s}$ ist auch der Einsatz als D4-Leim möglich. Bei einer Verleimung mit PH-Leim werden diese Anforderungen ebenfalls erfüllt. Eine Plasmabehandlung von $120 \mathrm{~s}$ führt hier zu Zugscherfestigkeiten entsprechend der EN 301 an tragende Holzkonstruktionen.

In wechselfeuchten Einsatzbereichen (EN 301 2006, A3) genügt ausschließlich eine Verleimung wachsimprägnierter Buche mit EPI-Leim sowie eine PVAC-Verleimung mit vorhergehender Plasmabehandlung von 120 s den Anforderungen der EN 301 und EN 204 an einen D3-Leim.

Danksagung Die Autoren danken der Clariant Produkte Deutschland $\mathrm{GmbH}$ (Gersthofen) für die Bereitstellung der Wachse sowie die Finanzierung der Arbeit. Weiterhin wurde die Arbeit vom Bundesministerium für Bildung und Forschung gefördert (FKZ: 1708X08).

Open Access Dieser Artikel unterliegt den Bedingungen der Creative Commons Attribution Noncommercial License. Dadurch sind die nichtkommerzielle Nutzung, Verteilung und Reproduktion erlaubt, sofern der/die Originalautor/en und die Quelle angegeben sind.

\section{Literatur}

Avramidis G, Nothnick E, Militz H, Viöl W, Wolkenhauer A (2010a) Accelerated curing of PVAc adhesive on plasma-treated wood veneers. Eur J Wood Wood Prod. doi:10.1007/s00107-010-0429-7

Avramidis G, Scholz G, Nothnick E, Militz H, Viöl W, Wolkenhauer A (2010b) Improved bondability of wax-treated wood following plasma treatment. Wood Sci Technol. doi:10.1007/s00226010-0327-5

Borgin K, Corbett K (1970) The stability and weathering properties of wood treated with various waxes. Paint Rubber 14:69-72

DGF (1975) DGF-Einheitsmethoden. Deutsche Gesellschaft für Fettwissenschaft, Abteilung M: Wachse und Wachsprodukte, Stuttgart

EN 204 (2001) Klassifizierung von thermoplastischen Holzklebstoffen für nichttragende Anwendungen. Beuth, Berlin

EN 301 (2006) Klebstoffe für tragende Holzbauteile. Phenoplaste und Aminoplaste. Klassifizierungs- und Leistungsanforderungen. Beuth, Berlin

EN 302-1 (2004) Klebstoffe für tragende Holzbauteile. Prüfverfahren. Teil 1: Bestimmung der Längszugscherfestigkeit. Beuth, Berlin
Feist WC, Mraz EA (1978) Protecting millwork with water repellents. For Prod J 28:31-35

Fengel D, Wegener G (2003) Wood. Chemistry, ultrastructure, reactions. Kessel, Remagen

Harzmann LJ (1988) Kurzer Grundriß der allgemeinen Tropenholzkunde. Hirzel, Leipzig

Illmann G, Schmidt H, Brotz W, Michalczyk G, Payer W, Dietsche W, Hohner G, Wildgruber J (1983) Wachse. In: Bartholomé E, Ullmann F (Hrsg) Ullmanns Enzyklopädie der technischen Chemie. Chemie. Wachse bis Zündhölzer, vol 24. Wiley-VCH, Weinheim

Kurt R, Krause A, Militz H, Mai C (2008) Hydroxymethylated resorcinol (HMR) priming agent for improved bondability of waxtreated wood. Holz Roh- Werkst 66:333-338

Militz H, Krause A (2008) Einsatz von modifiziertem Holz zur Verbesserung der Eigenschaften von Holzfenstern. Abschlussbericht DBU-AZ 22362

Nussbaum RM (1999) Natural surface inactivation of Scots pine and Norway spruce evaluated by contact angle measurements. Holz Roh- Werkst 57:419-424

Owens DK, Wendt RC (1969) Estimation of the surface free energy of polymers. J Appl Polym Sci 13:1741-1747

Scholz G, Krause A, Militz H (2009) Capillary water uptake and mechanical properties of wax soaked Scots Pine. In: Englund F, Hill CAS, Militz H, Segerholm BK (Hrsg) Proceedings of the fourth European conference on wood modification, Stockholm, Sweden, S 209-212

Scholz G, Krause A, Militz H (2010a) Beeinflussung der Holzfestigkeit durch Wachstränkung. Holztechnologie 51(3):22-27

Scholz G, Krause A, Militz H (2010b) Exploratory study on the impregnation of Scots pine sapwood (Pinus sylvestris L.) and European beech (Fagus sylvatica $\mathrm{L}$.) with different hot melting waxes. Wood Sci Technol. doi:10.1007/s00226-010-0353-3

Sell J (1977) Hydrophobierende Holzimprägnierung - Wirkungsweise, Eigenschaften, Anwendungsmöglichkeiten. Holz Roh- Werkst 35:75-78

Suchsland O (1958) Über das Eindringen des Leimes bei der Holzverleimung und die Bedeutung der Eindringtiefe für die Fugenfestigkeit. Holz Roh- Werkst 16:101-108

Wolkenhauer A (2009) Plasma treatment of wood and wood-based materials by dielectric barrier discharge at atmospheric pressure. Dissertation, Georg-August-Universität Göttingen, Sierke, Göttingen

Wolkenhauer A, Militz H, Viöl W (2008a) Increased PVA-glue adhesion on particle board and fibre board by plasma treatment. Holz Roh- Werkst 66:143-145

Wolkenhauer A, Avramidis G, Militz H, Viöl W (2008b) Plasma treatment of heat treated beech wood-investigation on surface free energy. Holzforschung 62:472-474

Wolkenhauer A, Avramidis G, Hauswald E, Militz H, Viöl W (2008c) Plasma treatment of wood-plastic composites to enhance their adhesion properties. J Adhes Sci Technol 22:2025-2037

Wolkenhauer A, Avramidis G, Hauswald E, Militz H, Viöl W (2009) Sanding vs plasma treatment of aged wood: a comparison with respect to surface energy. Int J Adhes Adhes 29:18-22 